\title{
Barrier RF Stacking
}

\author{
W. Chou ${ }^{1}$, D. Wildman ${ }^{1}$, H. Zheng ${ }^{2}$ and A. Takagi ${ }^{3}$ \\ ${ }^{1}$ Fermilab, USA, ${ }^{2}$ Caltech, USA, ${ }^{3}$ KEK, Japan
}

\begin{abstract}
A novel wideband RF system, nicknamed the barrier RF, has been designed, fabricated and installed in the Fermilab Main Injector. The cavity is made of seven Finemet cores, and the modulator made of two bipolar high-voltage fast solid-state switches. The system can deliver $\pm 7 \mathrm{kV}$ square pulses at $90 \mathrm{kHz}$. The main application is to stack two proton batches injected from the Booster and squeeze them into the size of one so that the bunch intensity can be doubled. High intensity beams have been successfully stacked and accelerated to $120 \mathrm{GeV}$ with small losses. The problem of large longitudinal emittance growth is the focus of the present study. An upgraded system with two barrier RF cavities for continuous stacking is under construction. This work is part of the US-Japan collaborative agreement.
\end{abstract}

Keywords: Barrier rf, longitudinal stacking, high intensity protons

\section{INTRODUCTION}

There are two major experimental programs at Fermilab at this time. One is Tevatron collider program Run2. Another is neutrino program including MiniBooNE ( $8 \mathrm{GeV}$ protons from the Booster for neutrino production) and NuMI (120 GeV protons from the Main Injector for neutrino production.). In these programs, a key technical parameter is the proton beam intensity. The more protons we have, the more anti-protons and neutrinos we can generate. However, there is a bottleneck in the present accelerator complex, namely, the Booster. It is a machine more than 30 years old and has never been upgraded. Even with significant improvements in the past several years, it can only deliver about 5E12 protons per cycle. The Main Injector, on the other hand, is a new machine and capable to accelerate much more protons than what the Booster can deliver. A logical solution, therefore, is to stack Booster beams in the Main Injector to increase the beam intensity. This is possible because the longitudinal acceptance of the Main Injector (about $0.7 \mathrm{eV}$-s) is larger than the Booster beam emittance (about $0.13 \mathrm{eV}-\mathrm{s}$ ). There are two stacking schemes that Fermilab is working on. One is slip stacking, [Ref. 1] another barrier rf stacking. [Ref. 2-4] This paper will describe the progress on the latter.

There are two methods to carry out barrier rf stacking. One is adiabatic stacking. In this case, the beam is injected on the energy axis in the longitudinal phase space. It is then debunched, slowly compressed by moving the $\mathrm{rf}$ barriers, recaptured by the main $\mathrm{rf}$ $(53 \mathrm{MHz}$ ) and accelerated to $120 \mathrm{GeV}$. Another is fast stacking, in which the beam is continuously injected with a small energy error (i.e., off the energy axis) and folded by two rf barriers - one stationary, another moving - such that twice as many Booster batches can be injected into the Main Injector and the beam intensity is doubled. The second method was first proposed by J. Griffin. [Ref. 5]

\section{BARRIER RF SYSTEM}

The barrier rf system consists of a cavity (figure 1) and a modulator (Figure 2). The cavity is made of seven Finemet cores. Finemet is a nanocrystal magnetic alloy patented by Hitachi. It has high magnetic permeability and low $\mathrm{Q}$ in the $\mathrm{MHz}$ range and is especially suitable for our application. Figure 3 shows a Finemet core. Figure 4 is a performance comparison between Finemet and ferrite 4M2. It is seen that Finemet can generate better square pulses than 4M2. The specifications of the Finemet cores are listed in Table 1 . The main components of the modulator are a pair of bipolar high voltage fast switches. They are made by Behlke Company in Germany. Figure 5 shows one of the two switches. The specifications are listed in Table 2. This system is similar to an rf chopper used at HIMAC in Japan. [Ref. 6-8] 


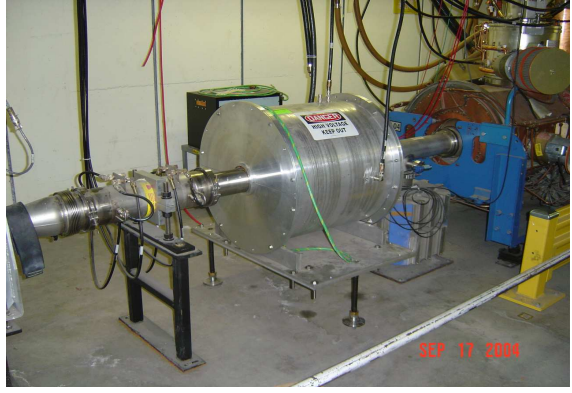

FIGURE 1. A barrier rf cavity in the Main Injector.

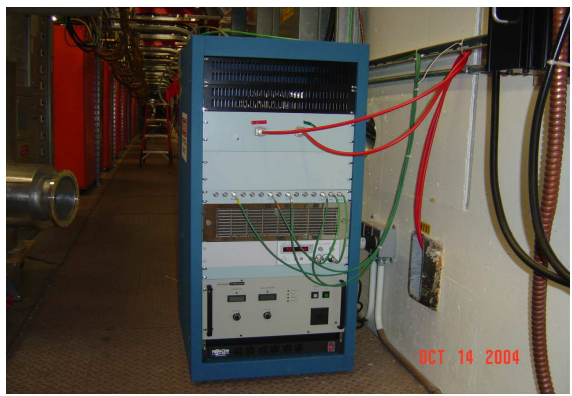

FIGURE 2. A barrier rf modulator.

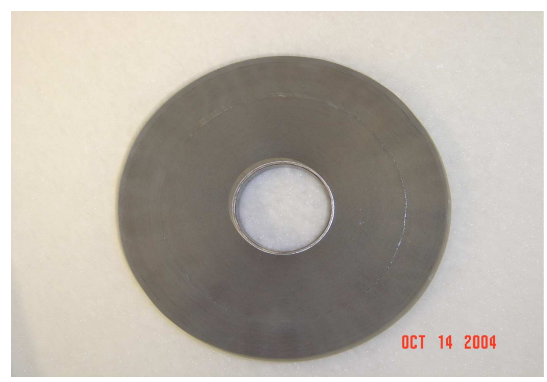

FIGURE 3. A Finemet core FT3M made by Hitachi.
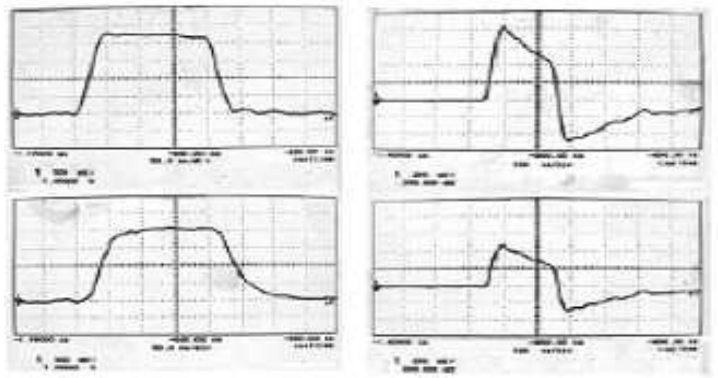

FIGURE 4. Performance comparison: Left -Finemet cores. Right $-4 \mathrm{M} 2$ cores. Upper ones are the primary voltage from the generator, lower ones the secondary voltage at the acceleration gap.

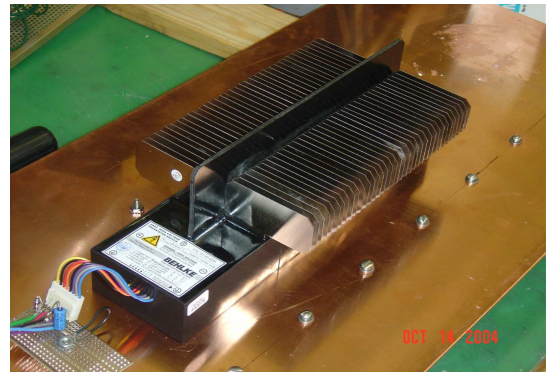

FIGURE 5. A high voltage solid-state fast switch HTS 16106-GSM made by Behlke.

TABLE 1. Finemet core specifications

\begin{tabular}{lc}
\hline Type & FT3M \\
\hline OD & $500 \mathrm{~mm}$ \\
ID & $139.8 \mathrm{~mm}$ \\
Thickness & $25 \mathrm{~mm}$ \\
Inductance & $56 \mu \mathrm{H}$ \\
Resistance & $190 \Omega$ \\
\hline
\end{tabular}

TABLE 2. High voltage fast switch specifications

\begin{tabular}{lc}
\hline Type & HTS $161-06-\mathrm{GSM}$ \\
\hline Peak voltage & $2 \times 16 \mathrm{kV}$ \\
Peak current & $60 \mathrm{~A}$ \\
Pulse width & $300 \mathrm{~ns}$ \\
Pulse spacing & $0.4-11 \mu \mathrm{s}$ \\
Repetition rate & $90 \mathrm{kHz}$ \\
Burst length & $400 \mathrm{~ms}$ \\
Burst repetition rate & $0.5 \mathrm{~Hz}$ \\
\hline
\end{tabular}

A particular concern in the design is radiation damage to the switches. The cavity is an inductive load, which would generate "ringing" after a single pulse. In order to minimize the ringing, the switches should be close to the cavity. However, the radiation level in the tunnel is high during machine operation, which could destroy the switches in a short time. Therefore, the switches must be far away from the beam line, which would lead to impedance mismatch between the load and the cable. A solution was found by using two transformers, one between the switches and the cable, another between the cable and the cavity. Figure 6 shows a complete circuit diagram for this system. The left part is the modulator, which is located in the rf gallery upstairs, and the right part is the equivalent circuit of the cavity, which is in the tunnel. Both are connected by 90 -feet long cables. 


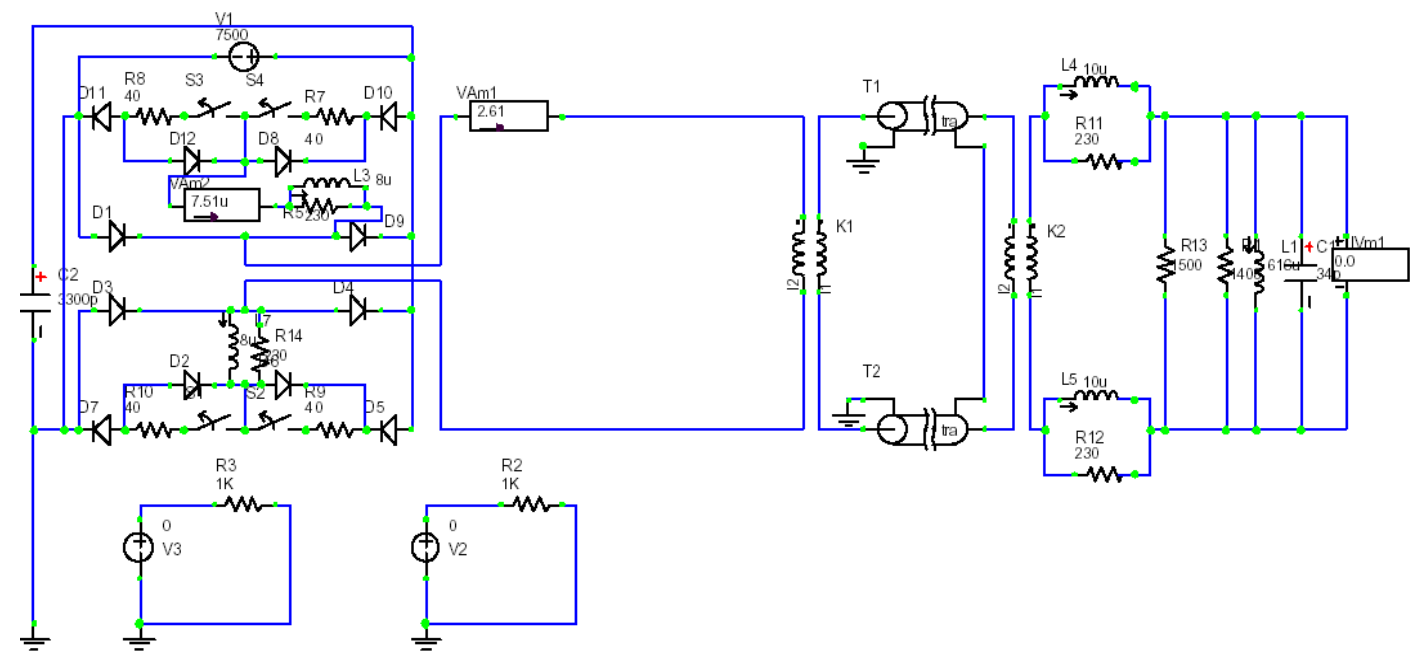

FIGURE 6. Circuit diagram of the barrier rf system. The left part is a modulator in the rf gallery, the right part a cavity in the tunnel. Both are connected by 90 -feet long cables.

Figure 7 shows the primary and secondary voltage pulses measured on a bench test. Due to stray capacitance in the system, one needs to pay special attention to the peak current and make sure it will not exceed the spec (60 A). Otherwise the switches could be destroyed and not be repairable.

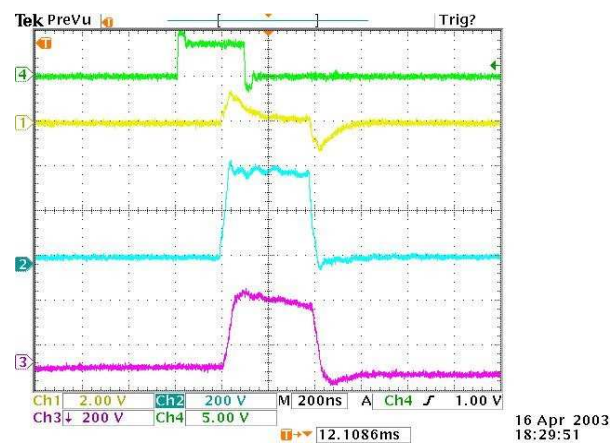

FIGURE 7. Bench test - from top to bottom: trigger signal (green), current (yellow), primary voltage (ligh blue) and secondary voltage (purple).

\section{BEAM EXPERIMENTS}

After the installation of this system in the Main Injector in the summer of 2004, a series of beam experiments were carried out. The procedure is described as follows.

Two consecutive Booster batches are injected into the Main Injector and captured by the $53 \mathrm{MHz}$ rf buckets. Each batch has 84 bunches for a length of 1.6 $\mu \mathrm{s}$. The two batches occupy a total length of $3.2 \mu \mathrm{s}$, which is $2 / 7$ of the Main Injector circumference. The $53 \mathrm{MHz}$ rf is then gradually turned off and the barrier rf turned on. So the beam is debunched and confined by the rf barriers. The barriers are slowly moved to squeeze the beam to half of its original size, i.e., from $3.2 \mu \mathrm{s}$ to $1.6 \mu \mathrm{s}$. Then the $53 \mathrm{MHz}$ rf is on again to recapture the beam and start acceleration. Figure 8 is an illustration of this process.

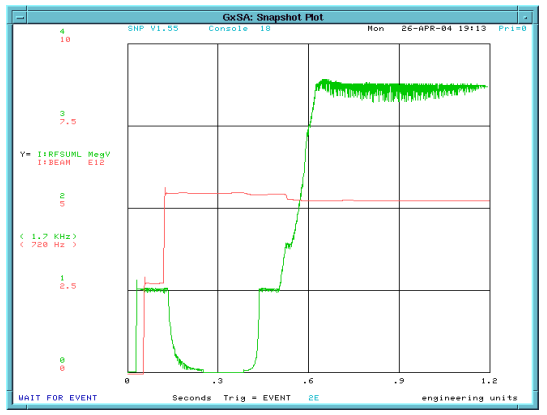

FIGURE 8. Barrier rf stacking process. Green $-53 \mathrm{MHz}$ rf curve, red - beam intensity. At the bottom of the green curve, the barrier rf is on and moves to squeeze the beam.

Figure 9 is a dynamic plot of the barrier rf voltage and the beam. It shows how the barriers move in and the beam gets squeezed. Figure 10 is a mountain view picture of the beam in the Main Injector. It shows clearly that the beam is squeezed to about half of its original size after stacking.

At the end of this process, the number of particles per bunch is twice as many as in the Booster beam. Figure 11 shows a beam of $8.3 \mathrm{E} 12$ is successfully accelerated to $120 \mathrm{GeV}$. This should be compared with 
a normal Booster beam of 5E12, an increase in intensity of about $60 \%$.

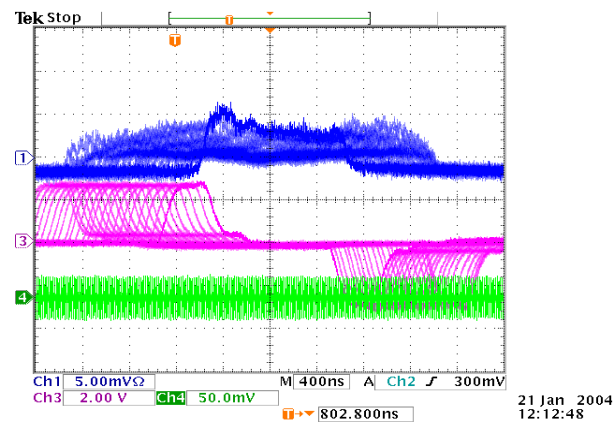

FIGURE 9. Dynamic plot of barrier rf voltage (middle curve in purple) and beam (top curve in blue). When the barriers move in, the beam gets squeezed.

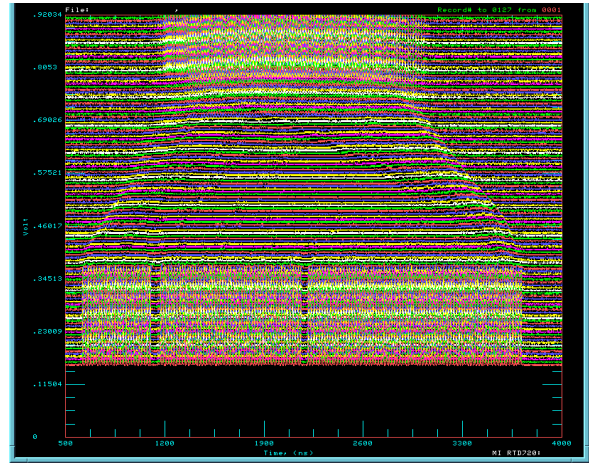

FIGURE 10. Mountain view picture of the beam. The vertical axis is time. The beam is squeezed to half size by moving the rf barriers.

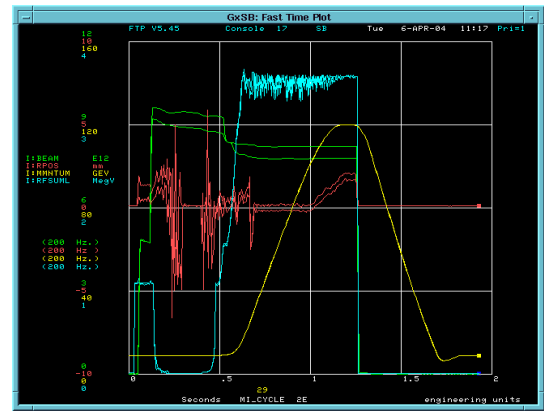

FIGURE 11. The green curves are the beam intensity during the cycle. After stacking, 8.3E12 protons are accelerated to $120 \mathrm{GeV}$, which is $60 \%$ higher than that in a normal Booster beam.

\section{DISCUSSION}

The barrier rf system works to our satisfaction. The beam experiments are successful. They demonstrate that barrier rf stacking is a feasible way to increase beam intensities in a machine. The whole process takes less than $0.5 \mathrm{sec}$ and can become part of the antiproton production cycle without increasing the cycle time $(2 \mathrm{sec})$

Before implementing this scheme in Run2, however, the longitudinal emittance dilution problem will have to be addressed. The beam loss in Figure 11 (a big drop in the green curve) is due to scraping at the beginning of acceleration when the $\mathrm{rf}$ bucket size is shrunk.

Figure 12 shows the beam size after stacking. At high intensity, the emittance dilution is significant.
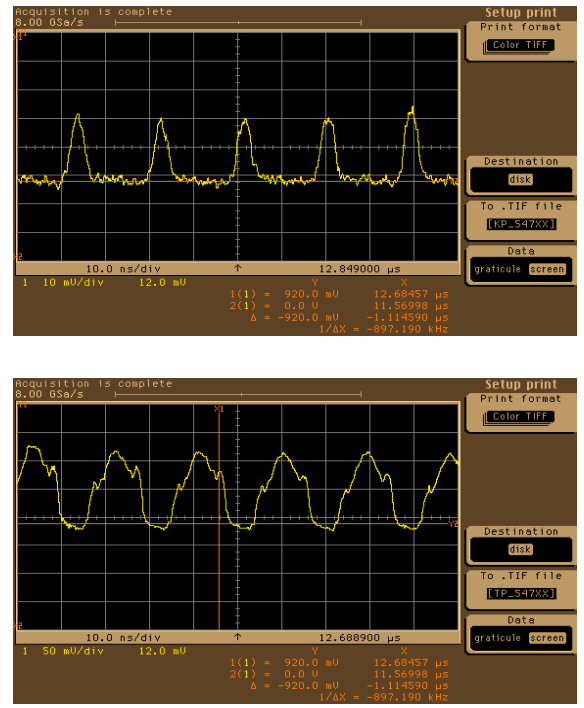

FIGURE 12. Beam spectrum after stacking: top - low intensity; bottom - high intensity.

A careful measurement gives $0.72 \mathrm{eV}$-s per bunch for a stacked high intensity beam at the early stage of acceleration (before transition). While a beam with such size can still cross the transition with almost no loss, it is too big for anti-proton production.

A possible source for the emittance dilution is beam loading. When the beam sits in between two barriers with the $53 \mathrm{MHz}$ rf off, beam loading would cause energy tilt from beam head to tail. Such a tilted beam would lead to larger emittance growth than does a leveled beam. A possible cure is to use the 
longitudinal damper in the Main Injector. This is the focus of our present study.

Another application of the barrier rf stacking is for NuMI during multi-batch operation. In this application, longitudinal emittance dilution is not as critical as in Run2. It would need two barrier rf systems, one stationary and another moving. We are in the process of fabricating a second barrier rf system and will install it in the Main Injector in 2005. When this system is in place, we will be able to employ the fast stacking method to accumulate 12 Booster batches in the Main Injector. (The normal operation calls for 6 batches.)

\section{ACKNOWLEDGMENTS}

In the past several years, a number of people have made contributions to this study, including J. Griffin, J. MacLachlan, K-Y. Ng, J-F. Ostiguy, C. Bhat, I. Kourbanis, Y. Mori and D. Michael. The authors would like to express their gratitude to these colleagues.

This work is partly supported by the US-Japan collaboration on high intensity proton $R \& D$.

\section{REFERENCES}

1. K. Koba and J. Steimel, AIP Conference Proc. 642, p. 223 (2002).

2. http://www-bd.fnal.gov/pdriver/barrier/

3. W. Chou et al., Proc. 2003 PAC (Portland, USA), p. 2922 (2003).

4. K.Y. Ng, AIP Conference Proc. 642, p. 226 (2002); also see FERMILAB-FN-715, FERMILAB-TM-2183 (2002).

5. http://www-bd.fnal.gov/pdriver/barrier/pdf/griffin.pdf

6. W. Chou et al., KEK Report 98-10 (September 1998); W. Chou et al., Proc. 1999 PAC (New York, USA), p. 565.

7. Y. Shirakabe et al., Proc. 2000 EPAC (Vienna, Austria), p. 2468.

8. C. Ohmori et al., NIM A 526, 215-221 (2004). 